\title{
Pancreatic Pseudocyst with Mediastinal Extension Presenting as Pseudo-Kirklin Sign-Multimodality Imaging
}

\author{
Harshini Udayakumar ${ }^{1}$ Venkatraman Indiran ${ }^{1} \quad$ Kalaichezhian Mariappan $^{1}$ \\ Prabakaran Maduraimuthu ${ }^{1}$
}

${ }^{1}$ Department of Radiodiagnosis, Sree Balaji Medical College and Hospital, Chennai, Tamil Nadu, India

\begin{abstract}
Address for correspondence Venkatraman Indiran, MD, DNB, Department of Radiodiagnosis, Sree Balaji Medical College and Hospital, 7 Works Road, Chromepet, Chennai, Tamil Nadu 600044, India (e-mail: ivraman31@gmail.com).
\end{abstract}

\begin{abstract}
Keywords

- Kirklin sign

- pancreatic pseudocyst

- chronic pancreatitis

A mass lesion of the gastric cardia or fundus causing an alteration in the normal regular, translucent gastric fundal air shadow on a frontal erect chest radiograph is referred to as "the Kirklin sign." Here we present "Pseudo-Kirklin sign" observed on the frontal radiograph of a 46-year-old male patient due to a soft tissue shadow/contour deformity of the fundal gas shadow caused by pseudocyst of the pancreas. We evaluated the patient using plain radiography, contrast enhanced computed tomography, magnetic resonance imaging, and endoscopic ultrasound (EUS) with the cyst drained under EUS guidance. So far only two cases of mediastinal pseudocysts have been drained successfully by EUS-guided aspiration.
\end{abstract}

\section{Introduction}

"The Kirklin sign" was described by radiologist Dr. B.R. Kirklin in his article on the radiologic features of cancer of the cardia, in 1939., ${ }^{1,2}$ Though Kirklin sign was classically coined for many malignant lesions of the fundus or cardia (gastric carcinoma, esophageal carcinoma, gastrointestinal stromal tumor), it has a range of differentials such as lymphoma, stromal cell tumor, gastroesophageal varices, and surgical procedures like fundoplication. ${ }^{2}$ Pancreatic pseudocyst causing Kirklin sign-like appearance on frontal plain chest radiograph has not been reported in literature so far. Despite the low probability of detecting a gastric carcinoma/pancreatic pathology on a chest radiograph, it is important to be aware of the importance of analyzing the gastric bubble for intraluminal/extraluminal pathologies.

\section{Case Report}

A 46-year-old male presented with complaints of abdominal pain, watery stools of increased frequency, weight loss, breathlessness, and generalized weakness for the past 15 days with no history of fever or vomiting. He was an alcoholic and smoker for the past 20 years. He was a diabetic patient for the past 5 years on irregular treatment and an old case of pulmonary tuberculosis.

Renal function test, complete blood count, and serum electrolytes were normal. Liver function test showed elevated levels of serum glutamic oxaloacetic transaminase $(168 \mathrm{mg} / \mathrm{dL})$, serum glutamic pyruvic transaminase (378 mg/dL), and gamma-glutamyl-transpeptidase (548 IU/L). Urine acetone was positive. Complete hemogram showed decreased hemoglobin of $12.2 \mathrm{mg} / \mathrm{dL}$. Erythrocyte sedimentation rate was elevated $(17 \mathrm{~mm})$. Elevated levels of serum amylase (859IU/L) and serum lipase (719 IU/L) were noted.

Plain erect radiograph of the chest in posteroanterior projection showed a nodular soft tissue density in the region of cardia producing the appearance of Kirklin sign ( - Fig. 1). Transabdominal ultrasonogram done in the supine position (on SIEMENS ACUSON S2000, Siemens Shangai Medica Equipment Ltd, China) showed a diffusely atrophic pancreatic parenchyma with a few calcific areas and a thick-walled cystic lesion arising from the pancreas, adjacent to the aorta and seen compressing the gastroesophageal junction ( - Fig. 2). No communication with the main pancreatic duct was noted. 


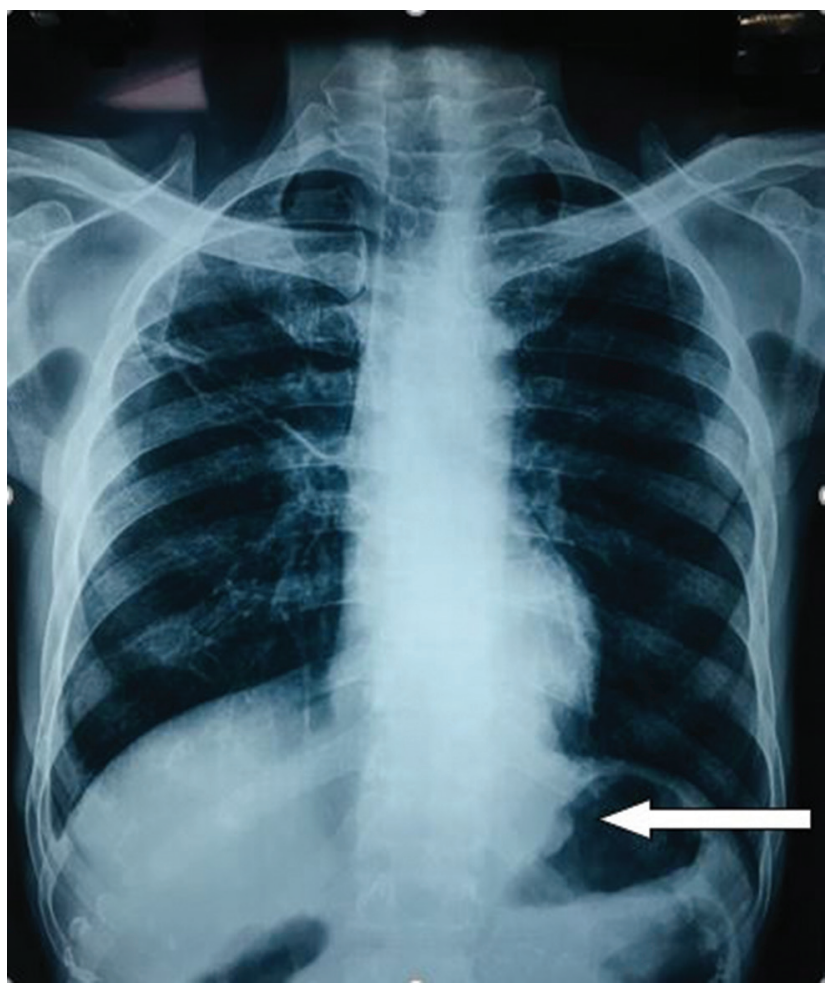

Fig. 1 Plain radiograph frontal projection shows an alteration in the normal regular, translucent gastric fundal air shadow (arrow).

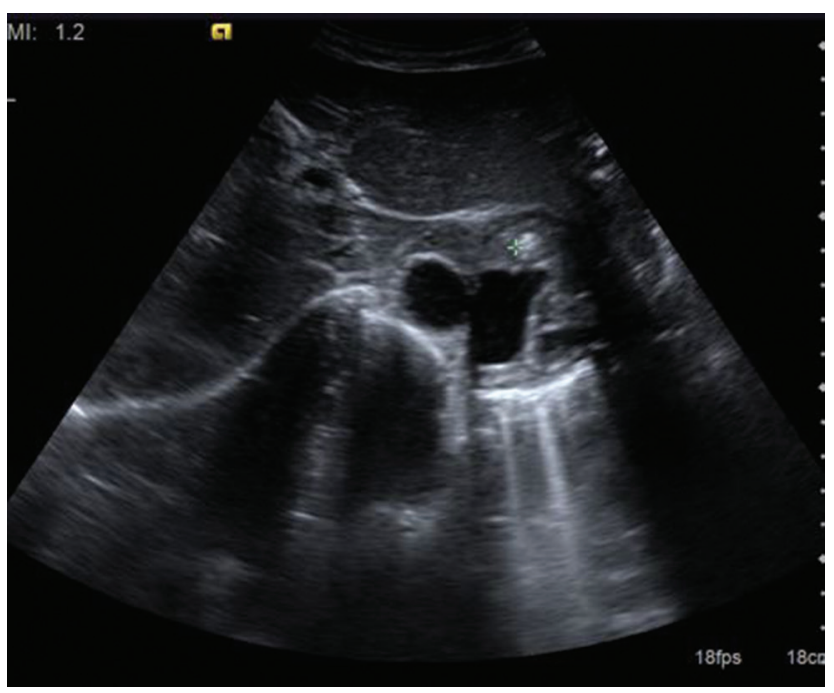

Fig. 2 Ultrasonogram of the abdomen showing a thick-walled cystic lesion (asterisk) adjacent to the aorta and compressing the gastro esophageal junction.

Gastroscopy showed edematous and congested mucosa was noted in the fundus and body.

Contrast enhanced computed tomography (CT) (Somotom Go Now (32 slice), Siemens Shangai Medica Equipment Ltd, China) was done with $1 \mathrm{~mL} / \mathrm{kg}$ bodyweight max view CT contrast agent with a flow rate of $3.0 \mathrm{~mL} / \mathrm{s}$. The pancreas appeared grossly atrophic with diffuse intra ductal/parenchymal calcifications. A large thick-walled organized collection measuring $\sim 8.0 \times 6.1 \times 7.3 \mathrm{~cm}$ was noted involving the paraesophageal, para-aortic region, perigastric regions and was seen tracking down into the lower surface of the tail of the pancreas suggestive of pancreatic pseudocyst with mediastinal extension ( - Fig. 3 ). No communication with the main pancreatic duct was noted. The visualized esophagus appeared compressed and collapsed. No significant lymph node enlargement or ascites was noted.

Magnetic resonance imaging $(\mathrm{T} 1, \mathrm{~T} 1+\mathrm{C}, \mathrm{T} 2$ on HITACHI APERTO 1.5T, Hitachi Medical Systems Ltd Sumpfstrasse 24,6300 Zug, Switzerland) showed a predominantly cystic lesion, $\mathrm{T} 1$ hypointense and $\mathrm{T} 2$ hyperintense with well-circumscribed wall, showing wall enhancement on contrast administration, located in the perigastric and paraaortic region (-Fig. 4).

Endoscopic ultrasound (EUS) of the cystic lesion provided us the option of cyst content aspiration and analysis as well as therapeutic drainage ( - Fig. 5). An elevated amylase level in the aspirated fluid (2790 IU/L) from the mediastinal pseudocyst confirms the diagnosis. The Gram stain showed no organisms. So, far only two cases of mediastinal pseudocysts have been drained successfully by EUS-guided aspiration. ${ }^{3}$

\section{Discussion}

Pancreatic pseudocysts, a common complication of both acute or chronic pancreatitis, account for $\sim 75 \%$ of all pancreatic masses. They may occasionally also extend into the mediastinum forming mediastinal pseudocysts with less than 100 cases reported in world literature. ${ }^{4}$

Pancreatic pseudocysts are cystic cavities encased by fibrous or reactive granulation tissue forming a well-defined wall distinguishing it from an acute fluid collection that can occur in the early course acute pancreatitis. ${ }^{5}$ Pancreatic enzymes, blood, and necrotic (dead) tissue form the content of the cyst.

Pancreatitis causing increased intraductal pressure due to ductal obstruction results in pseudocyst formation. It is believed that mediastinal pseudocysts are caused by pancreatic fluid riding up through the diaphragmatic openings, especially the esophageal and aortic openings being the most common entry sites into the posterior mediastinum. ${ }^{6}$ This is believed to be due to rupture of the pancreatic duct posteriorly into the retroperitoneal space. Pancreatic pseudocyst symptoms typically include abdominal pain with tenderness, diarrhea, fever, ascites, nausea and vomiting, weight loss, and jaundice. In some cases, pseudocysts can be asymptomatic. More than half of the pseudocysts spontaneously resolve, but they can also be complicated by intracystic bleeding, fistula formation, infection, increase in size and thus causing mass effect like bile duct or bowel obstructions. Conservative treatment measures and observation remain sufficient. If symptoms do not improve by 6 weeks, surgical intervention may be appropriate. ${ }^{7}$ Therefore, the mainstay of treatment involves avoiding the complication of rupture. As far as mediastinal pseudocysts are concerned, due to their rarity, the treatment measures are still evolving.

The CT scan showing a thin-walled cystic mass extending from the body of pancreas all the way into the mediastinum helped arrive at a definitive diagnosis. Contrast-enhanced CT 

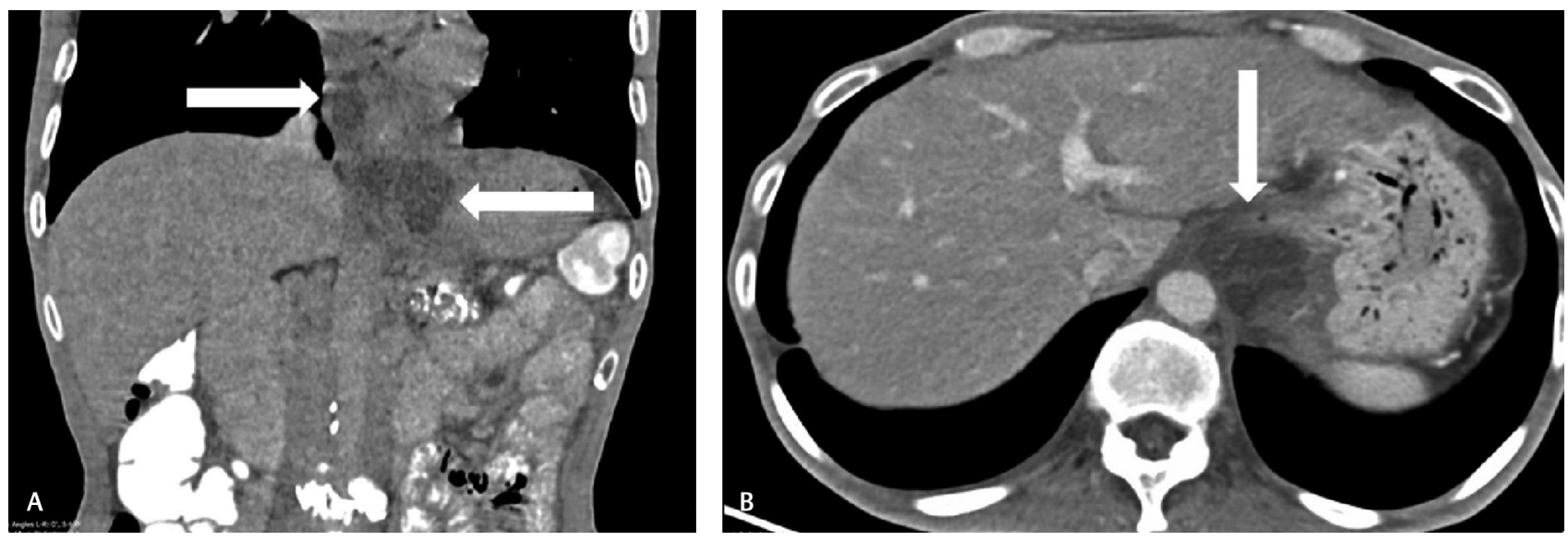

Fig. 3 Contrast-enhanced computed tomography abdomen in the portal venous phase (A) shows an atrophic pancreas with calcifications and a cystic lesion extending from the pancreas up into the mediastinum (arrow). Part (B) showed the perigastric and para-aortic location of the lesion (arrow).

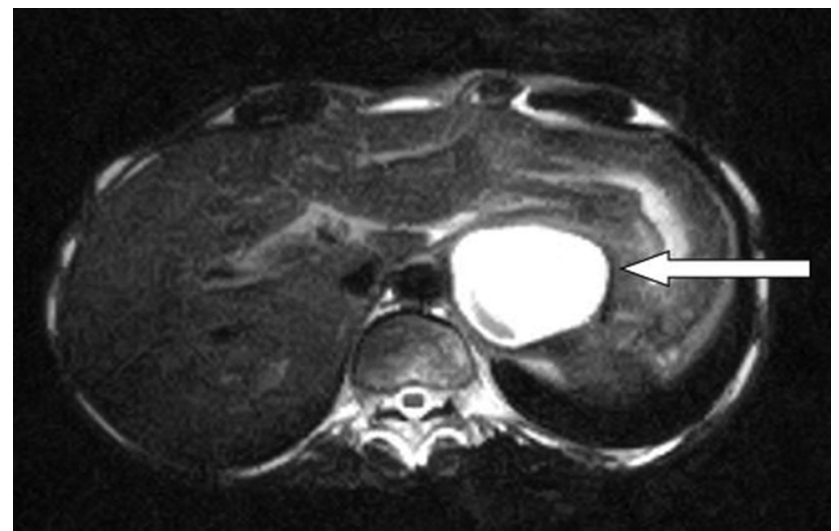

Fig. 4 Magnetic resonance imaging T2-weighted imaging axial section showing a hyperintense well-circumscribed, predominantly cystic lesion in the para-aortic and perigastric region (arrow).

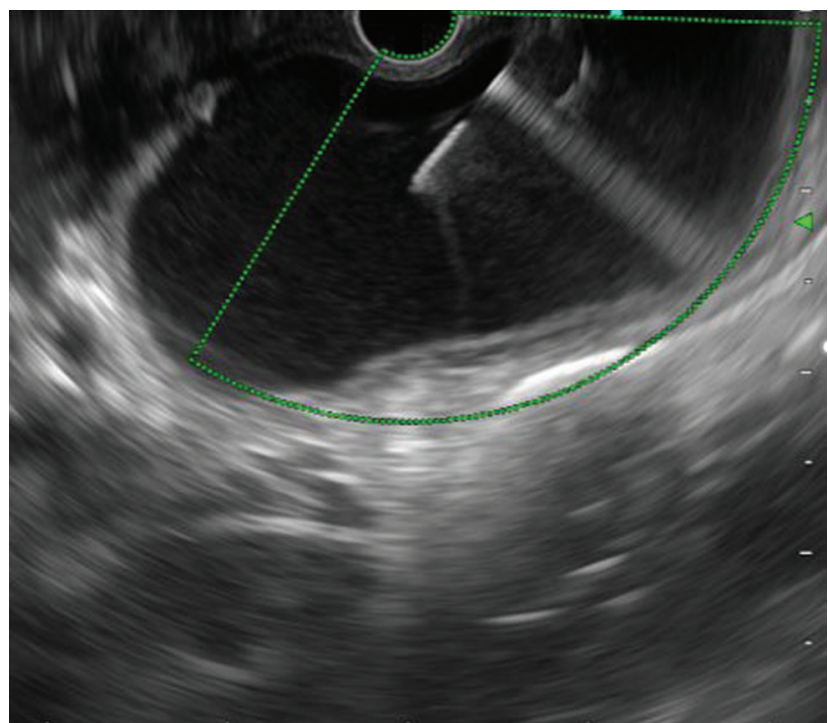

Fig. 5 Endoscopic ultrasound image demonstrating the pancreatic pseudocyst with $22 \mathrm{~g}$ fine-needle aspiration cytology needle in situ.

scan having a high sensitivity provided details regarding the anatomic location and relations of the pseudocyst with the neighboring structures.
A close differential diagnosis of pancreatic pseudocyst with mediastinal extension are proximal gastric/gastro esophageal pathologies. Gastroscopic examination remains the diagnostic method of choice and CT of the abdomen may reveal any carcinomas and help determine the invasion into adjacent tissues or the presence of spread to local lymph nodes. ${ }^{8}$ EUS is the recommended investigation for the evaluation of pseudocysts of the pancreas because it is both therapeutic and diagnostic. Additional information such as morphology of the cyst wall, characteristics of the fluid content, presence of gastric varices, collaterals, minimal peripancreatic fluid collection, distance between the gastric lumen, and the pseudocyst wall can be obtained. The fluid content influences the choice of stents. Anechoic pseudocysts require single drains and cysts with hypoechoic material (debris or necrosis) require multiple drains or nasocystic tubes. Cysts having focal wall thickening might impose the need for EUS-guided FNAC to clarify the diagnosis of the cystic neoplasms. Cysts having focal wall thickening might impose EUS-guided FNAC to clarify the diagnosis of the cystic neoplasms.

An elevated amylase level in the aspirated fluid from the mediastinal pseudocyst confirmed the diagnosis.

In cases where CT scan cannot delineate the pathology and help establish the abdominal and mediastinal connection of the mediastinal pseudocyst, magnetic resonance cholangiopancreatography can be useful. ${ }^{3}$

Hence, it is essential for the radiologist and gastroenterologists to be aware of this unusual imaging appearance of pancreatic pseudocyst with mediastinal extension producing a pseudo-Kirklin sign on frontal chest radiography, which has not been described so far in literature.

\section{Ethical Approval}

This article does not contain any studies with animals performed by any of the author(s). All procedures performed in studies involving human participants were in accordance with the ethical standards of the institutional and/or national research committee and with the 1964 Helsinki Declaration and its later amendments or comparable ethical standards. 


\section{Informed Consent}

Informed consent was obtained from all individual participants included in the study.

\section{Funding}

None.

\section{Conflict of Interest}

None declared.

\section{References}

1 Kirklin BR. Roentgenologic diagnosis of cancer of the cardia. Am J Radiol 1939;41:873-880

2 Indiran V, Kokilavani J, Ramachandra Prasad T, Kannan K. Kirklin sign. Abdom Radiol (NY) 2018;43(12):3505-3506
3 Gupta R, Munoz JC, Garg P, Masri G, Nahman NS Jr, Lambiase LR. Mediastinal pancreatic pseudocyst-a case report and review of the literature. Med Gen Med 2007;9(2):8

4 Dąbkowski K, Białek A, Kukla M, et al. Mediastinal pancreatic pseudocysts. Clin Endosc 2017;50(1):76-80

5 Habashi S, Draganov PV. Pancreatic pseudocyst. World J Gastroenterol 2009;15(1):38-47

6 Matsusue E, Fujihara Y, Maeda K, et al. Three cases of mediastinal pancreatic pseudocysts. Acta Radiol Open 2016;5(6):2058460116647213

7 Aghdassi AA, Mayerle J, Kraft M, Sielenkämper AW, Heidecke $\mathrm{CD}$, Lerch MM. Pancreatic pseudocysts-when and how to treat? HPB (Oxford) 2006;8(6):432-441

8 Virmani V, Khandelwal A, Sethi V, Fraser-Hill M, Fasih N, Kielar A. Neoplastic stomach lesions and their mimickers: spectrum of imaging manifestations. Cancer Imaging 2012;12:269-278 